\title{
QUO VADIS, ATHEROGENESIS? \\ PART 1. SMOOTH MUSCLE CELL SECRETION - HOW FOE BECOMES FRIEND IN THE FIGHT AGAINST THE ATHEROSCLEROTIC PLAQUE
}

\author{
Peter I. Ghenev', Luigi Aloe ${ }^{2}$, Antoniya R. Kisheva ${ }^{3}$, Manjinder Singh $^{4}$, \\ Plamen Panayotov ${ }^{5}$, Marco Fiore ${ }^{2}$, and George N. Chaldakov ${ }^{6 *}$ \\ ${ }^{1}$ Department of General and Clinical Pathology, Medical University, Varna, Bulgaria \\ ${ }^{2}$ Institute of Cell Biology and Neurobiology, National Research Council (CNR), Rome, Italy \\ ${ }^{3}$ Clinic of Cardiology, Department of Internal Medicine, St Marina University Hospital, Varna, Bulgaria \\ ${ }^{4}$ School of Pharmaceutical Sciences, Lovely Professional University, Phagwara, Punjab, India \\ ${ }^{5}$ Department of Cardiac Surgery, St Marina University Hospital, Varna, Bulgaria \\ ${ }^{6}$ Department of Anatomy and Cell Biology, Medical University, Varna, Bulgaria
}

\section{ABSTRACT}

Atherosclerosis is a chronic inflammatory disease in which exacerbation leads to myocardial infarction, stroke and/or lower limb ischemia. Phenotypic plasticity of artery smooth muscle cells (SMC) that can adapt to changes in the injured arterial microenvironment is a major determinant of atherosclerotic plaque vulnerability. Plaque instability has been associated with the ulceration or rupture of the fibrous cap composed primarily of SMC and collagen and elastin fibers, that covers the lipid core of the plaque. In this scenario, we, together with SMC, Dance round recent advances that have shed light on the relationship between inflammation, fibrosis and plaque vulnerability and stability. Specifically, we have addressed the question of how the secretory (fibrogenic) activity of SMC occurring within the plaque may become a plaque stabilizer (a friend). We describe a new paradigm shift in the cell biology of atherosclerosis that relates the inhibition of SMC matrix secretion and proliferation (the classical way for reducing plaque size) to the stimulation of these processes (the new way aimed at the plaque stabilization by increasing the thickness of its fibrous cap). Briefly, an increased secretion of matrix molecules, particularly collagen and elastin, by SMC could "shift" them from foe to friend in the fight against the vulnerable atherosclerotic plaque. Biomed Rev 2017; 28:134-138.

Keywords: atherosclerotic plaque, fibrous cap, smooth muscle cells, macrophages, phenotypic modulation, matrix proteins, inflammation, fibrosis, colchicine

\section{INTRODUCTION}

Whereas the precise nature of the initiating event for atherosclerosis is not known, it is clear that the failure of the smooth muscle cell to maintain its normal differentiated phenotypic state becomes a key contributing factor in the progression of atherosclerotic disease.

\section{Owens GK (20)}

Atherosclerosis is a chronic inflammatory fibroproliferative disease in which exacerbation leads to myocardial infarction, stroke and/or lower limb ischemia (1-9). It is the

Received 19 November 2017, revised 7 December 2017, accepted 8 December 2017

* Correspondence and reprint request to Dr George N. Chaldakov, Department of Anatomy and Cell Biology, Medical University, BG-9002 Varna, Bulgaria

E-mail: chaldakov@yahoo.com 
leading cause of morbidity and mortality worldwide. There is general consensus that the majority of acute coronary and cerebrovascular syndromes are the result of rupture or erosion of the thinner fibrous cap ( $<65$ micrometer) of unstable (vulnerable, culprit) plaques and associated thrombotic events. One of the biggest challenges in cardiology today is to identify the patient who is at risk for a future myocardial infarction, since most acute cardiac events occur without previous symptoms. According to histopathological studies, about $70 \%$ of myocardial infarctions are caused by plaque rupture and another $30 \%$ by plaque erosion (10). The fibrous cap is an atheroprotective layer presumed to be composed primarily of bundles of phenotypically modulated smooth muscle cells (SMC) and collagen and elastin fibers that cover the plaque's lipid core. The latter is featured by the presence of (i) large numbers of cells positive for macrophage markers, (ii) foam cells, and (iii) extracellular lipids.

These data are interpreted as evidence that atherosclerotic plaques with a preponderance of macrophages, lymphocytes and macrophage-derived foam cells relative to SMC, particularly within the fibrous cap and plaque's shoulder regions, are less stable and more prone to rupture. That is, SMC in advanced lesions are generally regarded as having atheroprotective plaque-stabilizing properties whereas macrophages and lymphocytes are viewed as being atheropromoting and detrimental for plaque stabilization. In brief, plaques which contain a high ratio of macrophages and lymphocytes relative to SMC are less stable, particularly those that have a thin fibrous cap (1-8).

Despite extensive research on the cell biology of atherosclerosis, there are still fundamental questions in regard to the transition of a plaque towards its ulceration, erosion, fissure and rupture. For instance, the resident SMC that constitute the majority of the native vessel wall undergo dramatic phenotypic changes in the process of atherogenesis, with the loss of SMC gene expression in the medial vessel layer and appearance of a new layer of $\alpha$-actin expressing SMC in a fibrous cap (5-7). While the risk of plaque rupture appears to be inversely correlated with the number of SMC in the fibrous cap, there is very little understanding of their in vivo origin, and the molecular pathways that regulate their phenotype switching (5-7). In brief, there is now compelling evidence that a full understanding of SMC modulation, function and origin are critical to identifying therapeutic targets for both prevention and therapy of atherosclerosis.

\section{THE SMOOTH MUSCLE CELL: THE PIVOT IN OCCLUSION VERSUS RUPTURE OF ATHEROSCLEROTIC PLAQUE}

Arterial SMC are critical contributors to atherosclerosis development. There is a dramatic alteration in gene expression and basic cellular functions that occur with phenotypic modulation of these cells in the disease setting $(5,6)$. In the classic, Russell Ross' response-to-injury hypothesis (reviewed in 1), fully differentiated medial SMC express contractile marker genes and have limited proliferative capacity, while under disease conditions they downregulate contractile gene expression, proliferate, and migrate into the neointimal space. It is generally accepted that SMC accumulation in the fibrous cap is monoclonal or oligoclonal, and this is consistent with the existence of a resident arterial subpopulation of SMC precursor cells that contribute to healing in the response to disease stresses.

Arterial SMC are distinguished from other cell types by expression of a unique repertoire of genes $(5,6)$. These cells have pleiotropic capacities of phenotypic modulation, one of them associated with the secretion of multiple matrix molecules as well as mediators of cell growth, migration, inflammation, and healing. Today, Ross' hypothesis, established in the early 1970 's, is still the prevailing dogma on atherogenesis. It states that "the lesions of atherosclerosis represent a protective, inflammatory fibroproliferative response" that involves several aspects of vascular wound healing [1; for nerve growth factor (NGF) in wound healing, see 8, 14]. The proliferation, migration and secretory activities of SMC are crucially implicated in this intimal "wound". Consequently, modulation of arterial SMC from contractile to secretory phenotype resulting in cell proliferation and matrix molecule oversecretion has held center stage for about three decades as the protagonist in plaque formation as well as angioplasty-associated neointimal hyperplasia. Thus the increased SMC proliferation/matrix production was considered as the major foe to be pursued (1-3, $8,9,11-13)$, as it causes high-grade arterial occlusion. So the initial understanding was that the gradual increase of plaque volume contributes to arterial blood flow reduction leading to clinical complications. Numerous studies, especially image analyzing methods revealed that the culprit lesion is rarely associated with significant arterial stenosis; usually it is medium sized plaque with thin fibrous cap and large lipid core, also known as the vulnerable plaque, and vulnerable patient respectively. The chronology of events and the relation between stable and vulnerable plaques is beyond of being fully clarified. In this volume of Biomedical Reviews we (9) have presented one of the innovative research projects from the Laboratory 
of Electron Microscopy (later renamed Lab of Cell Biology), Medical University, Varna, Bulgaria. This concerned the secretory function of arterial SMC, with special reference to cytoskeletal microtubules (MT) and Golgi-derived secretion granules (vacuoles), the secretory pathway being tested with colchicine treatment to disassemble MT accompanied by accumulation of secretory granules, suggestive of the inhibition of the secretory pathway. This latter effect might be considered antifibrotic, hence cautiously applicable to the inhibition of fibrogenesis featured in several diseases, including atherosclerosis and related disorders $(9,11-14)$.

Things changed dramatically in the late1990's. The historian Thomas Kuhn refers to such changes as a "paradigm shift". The attention to SMC was moved from the plaque-associated occlusion toward the integrity of plaque's fibrous cap as a critical factor for the plaque stability (1-3). And, respectively, from decreasing SMC proliferation/matrix production aimed at vascular occlusion decrease toward increasing SMC proliferation/matrix production aimed at plaque rupture prevention. Basic and clinical studies recognized that human atherosclerotic plaques prone to alterations involving fissuring, erosion, ulceration and rupture of the plaque surface (unstable plaques, "ulcerative intimitis") usually have fewer SMC and collagen fibers, numerous inflammatory cells, and a large lipid-rich core. This insufficiency in SMC/matrix forms a thin, vulnerable fibrous cap, while SMC/matrix excess improves the biomechanical strength of the fibrous cap, thus stabilizing the plaque surface (stable plaques, "fibrous intimitis"). Noteworthy, the decreased SMC proliferation and matrix production, rather than its increase, directly accounts for plaque complications, such as unstable angina and myocardial infarction (Table 1).

Table 1. Main morphological differences in stable versus unstable plaque

\begin{tabular}{|l|l|}
\hline \multicolumn{1}{|c|}{ Stable plaque } & \multicolumn{1}{c|}{ Unstable plaque* } \\
\hline Small lipid core & Large lipid core \\
\hline Thick fibrous cap & Thick fibrous cap \\
\hline Low microvessel density & High microvessel density \\
\hline Low macrophage content & High macrophage content \\
\hline No intraplaque hemorrhage & $\begin{array}{l}\text { Presence of intraplaque } \\
\text { hemorrhage }\end{array}$ \\
\hline No cap rupture, no thrombus & $\begin{array}{l}\text { Presence of cap rupture } \\
\text { and thrombus }\end{array}$ \\
\hline
\end{tabular}

* For viewing the atherosclerotic plaque as a vascular wound, see $(8,14)$. For anti-inflammatory therapy, see $(9,15-19)$.

\section{AN EXCITING STORY}

Reminding that "The new paradigm is not only different but also better..." (a quote from Thomas S. Kuhn's The Structure of Scientific Revolutions), we argue that paradigm shift is also valid in the atherogenic saga of SMC:

(i) in the 1960's, Maria Daria Haust has made the first paradigm shift in the field pointed out to a new, synthetic versus contractile function of the artery SMC,

(ii) in the 1970 's, we have published the first transmission electron microscopic (TEM) data of this new function conceptualizing it as "secretory function" instead of "synthetic function" of SMC as has been previously considered (reviewed in 12, 14),

(iii) in 1980's, atherosclerosis was rediscovered as an inflammatory disease (firstly suggested by the great Rudolf Virchow) due to the research work of Russell Ross, Peter Libby, Jack P. Strong and other scientists, and

(iv) in 1990's, the next paradigm shift has emerged, that is, the importance of SMC for the thickness of atherosclerotic plaque's fibrous cap relative to plaque stability and vulnerability (1-3).

This indeed makes an exciting story!

\section{CONCLUSION}

Until things change again, SMC appear to be a friend rather than a foe, since they may stabilize/heal the plaque, although they increase the occlusion grade (1-9). However, from the therapeutic standpoint, there stands a balance of having either too much (excessive occlusion) or too little (excessive vulnerability) of a "friend" of the artery? If the answer to this question holds any water, then by reinstructing both the SMS and the macrophages, the two main players in this scenario, one might develop (as is being attempted these days) new therapeutic approaches by giving the right signals to these cells to revert to their normal phenotype. For instance, it is know that inflammation is a two-step process - initiation and resolution. Upregulating this latter step may represent a "resolution pharmacology" approach (16 and references therein). One thing is clear: our views on atherogenesis are indeed changing, but much more debate is required to sort out a plausible basis that embraces most of these alternative mechanisms. Further work on molecular and cellular mechanisms 


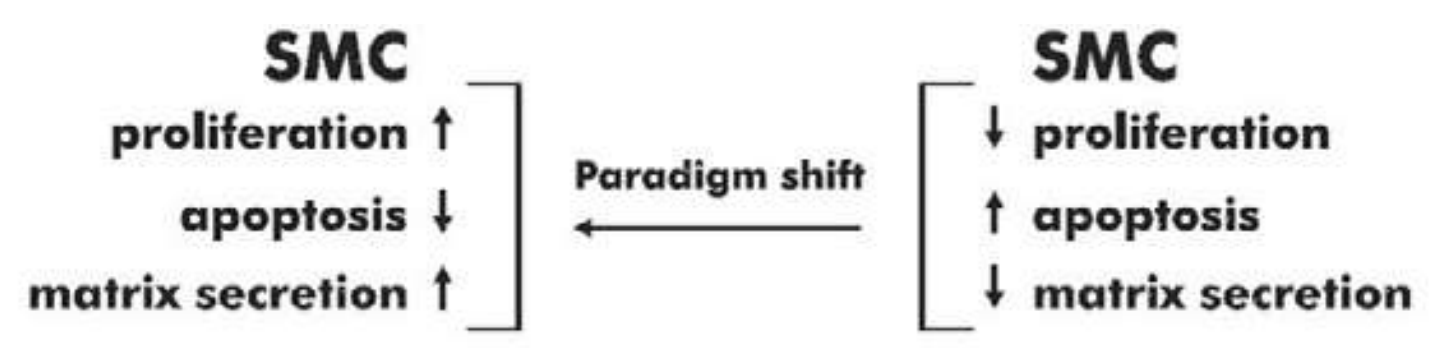

\section{Angioplasty}

Inflammation $\downarrow$ Lipids $\downarrow$

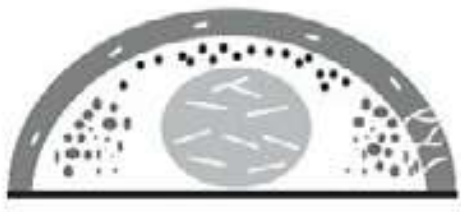

Unstable plaque

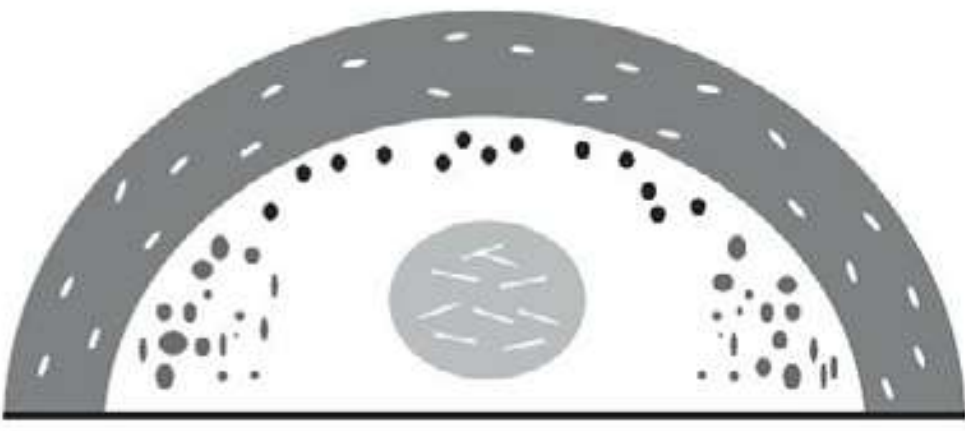

Stable plaque

Figure 1. A scheme showing possible ways to modulate atherosclerotic plaque development. The long arrow at the upper part indicates a paradigm shift in our knowledge of the atherogenesis-related potential of smooth muscle cells (SMC). That is, from the inhibition of SMC matrix secretion (e.g., by colchicine, see 11-15) and proliferation - the classical way for reducing plaque size -, to the stimulation of these processes (the new way aimed at increasing the thickness of fibrous cap). Thus an increased secretion of collagen and elastin by SMC could "shift" them from foe to friend in the fight against the unstable plaque. Depicted at the lower part of the figure are (i) unstable plaque shown at "a lower magnification", and (ii) stable plaque - at "a higher magnification" The bidirectional arrows mark the big question in plaque's fate, which is critical for the human life. From (8).

of wound-healing phenotypes, including scarring, keloid and ulceration, is required for the better understanding of intimabased vascular occlusion and fibrous cap-based plaque rupture $(1,8,14$ and references therein)

Of note, considering the importance of fibrous cap for the vulnerability of the plaque, we must proceed cautiously with antifibrotic [and antiproliferative although LoDoCo (low dose of colchicine) is presumably sub-antimitotic] action of colchicine, which could decrease the thickness of fibrous cap, thus making the plaque vulnerable to rupture (Fig. 1). Who knows, there may be other mechanism of atherogenesis waiting to be discovered. As Denys Wheatley wrote in his Eureka review published in this volume of Biomedical Reviews (21), we "do need to think outside the box; to paraphrase Schopenhauer": thus, the task is, not so much to see what no one has seen yet, but to think what nobody has thought yet, about what everybody sees."

\section{CONFLICT OF INTEREST STATEMENT}

The authors certify that they have no affiliations with or involvement in any organization with any financial interest in the subject matter discussed in this Dance round.

\section{ACKNOWLEDGEMENTS}

This Dance round could not be performed without the staunch support and creative collaboration over the years of our brainand-heart friends (BHF) Anna Kadar, Jörg Grünwald, Yukio Yamori, Toru Nabika, Takashi Fujiwara, Anton B. Tonchev and Stanislav Yanev. The linguistical improvement of the manuscript made by Professor Denys Wheatley (University 
of Aberdeen, UK) is greatly appreciated. We apologize to the authors of many relevant articles that have not been quoted here for reasons of brevity.

\section{REFERENCES}

1. Ross R. Mechanisms of disease: atherosclerosis - an inflammatory disease. $N$ Engl J Med 1999; 340: 115126.

2. Schwartz SM, Virmani R, Rosenfeld ME. The good smooth muscle cells in atherosclerosis. Curr Atheroscler Rep 2000; 2: 422-429.

3. Lafont A, Libby P. The smooth muscle cell: sinner or saint in restenosis and the acute coronary syndromes? $J$ Am Coll Cardiol 1998; 32: 283-285.

4. van Lammeren G, L Moll F, Borst GJ, de Kleijn DP, P M de Vries JP, Pasterkamp G, et al. Atherosclerotic plaque biomarkers: beyond the horizon of the vulnerable plaque. Curr Cardiol Rev 2011;7(1):22-27. DOI:10.2174/157340311795677680

5. Nurnberg ST, Cheng K, Raiesdana A, Kundu R, Miller CL, Kim LB, et al. Coronary artery disease associated transcription factor TCF21 regulates smooth muscle precursor cells that contribute to the fibrous cap. PLoS Genet 2015; 11(5): e1005155. DOI: 10.1371/journal. pgen. 1005155

6. Shankman LS, Gomez D, Cherepanova OA, Salmon M, Alencar GF, Haskins RM, et al. KLF4 dependent phenotypic modulation of SMCs plays a key role in atherosclerotic plaque pathogenesis. Nat Med 2015; 21(6): 628-637. DOI: 10.1038/nm.3866

7. Bennett MR, Sinha S, Owens GK. Vascular smooth muscle cells in atherosclerosis. Circ Res 2016; 118(4): 692-702. DOI: 10.1161/CIRCRESAHA.115.306361

8. Chaldakov GN, Fiore M, Ghenev PI, Stankulov IS, Angellucci F, Pavlov PS, et al. Conceptual novelties in atherogenesis: Smooth muscle cells, adventitia, and adipose tissue. Biomed Rev 2000; 11: 63-67.

9. Chaldakov GN, Ghenev PI. Colchicine, inflammation and fibrosis in cardiovascular disease: Merging three classical tales. Biomed Rev 2017; 28: 110-115.

10. Pflederer T, Marwan M, Schepis T, Ropers D, Seltmann M, Muschiol G, et al. Characterization of culprit lesions in acute coronary syndromes using coronary dualsource CT angiography. Atherosclerosis 2010; 211:437444.DOI:10.1016/j.atherosclerosis.2010.02.001

11. Chaldakov GN. Antitubulins - a new therapeutic ap- proach for atherosclerosis? Atherosclerosis 1982; 44: 385-390.

12. Chaldakov GN. George E. Palade lecture. Human body as a multicrine system, with special reference to cell protein secretion: From vascular smooth muscles to adipose tissue. Biomed Rev 2016; 27:VIII-XIX.

13. Chaldakov GN, Deyl Z, Vankov VN. Colchicine: possible new application of its antifibrotic (antisecretory) action. Physiol Bohemoslov 1987;36:1-7.

14. Yanev S, Fiore F, Hinev A, Ghenev PI, Hristova MG, Panayotov $\mathrm{P}$, et al. From antitubulins to trackins. Biomed Rev 2016; 27: 59-67.

15. Solak Y, Siriopol D, Yildiz A, Yilmaz MI, Ortiz A, Covic A, et al. Colchicine in renal medicine: New virtues of an ancient friend. Blood Purif 2017;43:125135. DOI: 10.1159/000454669.

16. Sansbury BE, Spite M. Resolution of acute inflammation and the role of resolvins in immunity, thrombosis and vascular biology. Circ Res 2016; 119(1): 113-130. DOI: 10.1161/CIRCRESAHA.116.307308

17. Martinez GJ, Robertson S, Barraclough J, Xia Q, Mallat Z, Bursill C, et al. Colchicine acutely suppresses local cardiac production of inflammatory cytokines in patients with an acute coronary syndrome. J Am Heart Assoc 2015;4:e002128. DOI: 10.1161/ JAHA.115.002128.

18. Robertson S, Martinez GJ, Payet CA, Barraclough JY, Celermajer DS, Bursill C, et al. Colchicine therapy in acute coronary syndrome patients acts on caspase- 1 to suppress NLRP3 inflammasome monocyte activation. Clin Sci (Lond) 2016; 130:1237-1246. DOI: 10.1042/ CS20160090.

19. Ridker PM, Howard CP, Walter V, Everett B, Libby P, Hensen J, et al; Group CPI. Effects of interleukin-1beta inhibition with canakinumab on hemoglobin A1c, lipids, C-reactive protein, interleukin-6, and fibrinogen: a phase IIb randomized, placebo-controlled trial. Circulation 2012;126: 2739-2748.

20. Owens GK. Role of alterations in the differentiated state of smooth muscle cell in atherogenesis. In: Fuster $\mathrm{V}$, Ross R, Topol EJ, editors. Atherosclerosis and Coronary Artery Disease. Philadelphia - New York. Lippincott-Raven, 1996; 1:401-420.

21. Wheatley D. On the nature of scientific discovery and progress - lessons from a career in cancer research. Biomed Rev 2017; 28:116-125. 